\title{
FUNCTION SPACES CONTINUOUSLY PAIRED BY OPERATORS OF CONVOLUTION-TYPE
}

\author{
BY \\ R. A. KERMAN
}

\begin{abstract}
Certain operators essentially defined by convolution are considered. Their possible domain and range spaces are determined; then conditions are given under which the construction of the optimal continuous partner may be carried out for a suitable domain or range. Special cases of operators of convolution-type are useful in studying the boundedness properties of conjugate function operators and, more generally, classes of operators satisfying restricted weak-type conditions.
\end{abstract}

1. Introduction. In this paper we fix on a positive operator $T$ of convolution-type and give conditions under which one can construct with respect to it an optimal continuous partner for a proposed domain or range. Such a $T$ has the form

$$
(T f)(t)=\int_{0}^{\infty} a(s) f(s t) d s, \quad t>0 ;
$$

the domain consists of all functions $f$ in the class of Lebesgue-measurable functions on $(0, \infty)$, denoted by $M(0, \infty)$, for which the integral exists a.e.; the kernel $a(t)$ is a nonnegative function in $M(0, \infty), a(t) \neq 0$. Motivation for the term "convolution-type" may be found in [5] and references cited there.

It has been shown that the boundedness of certain conjugate function operators between a pair of rearrangement invariant function spaces is equivalent to that of a $T$ with kernel

$$
\min \left[t^{1 / p-1}, t^{1 / q-1}\right] \quad 1 \leq p<q \leq \infty .
$$

The first theorem of this kind was proved in Boyd [3] for the Hilbert transformation. Further results and references are given in [7]. Such operators also play a special role in the theory of operators of restricted weak-type. See Calderón [6]-in particular the discussion of optimal pairs in section 3-and Boyd [4]; also [8].

Theorems 2.2 and $2.2^{\prime}$ give the conditions for the construction of optimal continuous partners. These apply, in particular, to $T$ having kernels (1.2). The continuous pairs thus determined are the same as those for the conjugate function operators mentioned above.

Received by the editors July 8, 1977 and, in revised form, April 20, 1978.

Research supported in part by NRC Grant \#A4021. 
As shown in Theorem 2.1 there is a maximum domain and a minimum range for the $T$ of the above two results; Theorems 2.3 and $2.3^{\prime}$ describe their optimal partners when $T$ has a kernel (1.2).

Background material on rearrangement invariant spaces and convolutiontype operators may be found in [4] and [5]. We will use the notation $[X, Y]$ for the space of linear operators bounded from $X$ to $Y$, abbreviating $[X, X]$ by $[X]$. Finally, if $T$ is a positive operator of convolution-type with kernel $a(t)$, the operator $T^{\prime}$ with kernel $(1 / t) a(1 / t)$ will be called its associate operator if

$$
\int_{0}^{\infty} f(t)(T g)(t) d t=\int_{0}^{\infty} g(t)\left(T^{\prime} f\right)(t) d t
$$

for all nonnegative $f, g \in M(0, \infty)$.

2. Continuous Pairs. If a postitive $T$ of convolution-type is in $[X, Y], X$ and $Y$ being rearrangement invariant with respect to Lebesgue's measure $m$ on $(0, \infty)$, then $T \chi_{(0,1)}$ must be locally integrable. It is then a consequence of Theorem 2.1 below that should $T$ be bounded between a pair of rearrangement invariant spaces there will exist two Lorentz spaces, one of which is the largest possible domain space for $T$; the other, the smallest possible range space. As is well-known, given a nonegative, nonincreasing function $\phi$ on $(0, \infty)$ with

$$
\Phi(t)=\int_{0}^{t} \phi(u) d u<\infty, \quad t>0
$$

the (rearrangement invariant) Lorentz spaces $\Lambda(\phi)$ and $M(\phi)$ have their norms given at nonnegative $f \in M(0, \infty)$ by

$$
\sigma(f)=\int_{0}^{\infty} f^{*}(t) \phi(t) d t
$$

and

$$
\sigma^{\prime}(f)=\sup _{t>0} \int_{0}^{t} f^{*}(u) d u / \Phi(t),
$$

respectively. Here $f^{*}$ is the nonincreasing rearrangement of $f$. Further, as the notation in (2.2) suggests, $M(\phi)$ is the space associate to $\Lambda(\phi)$.

THEOREM 2.1. Suppose $T$ is a positive operator of convolution-type with associate $T^{\prime}$. Let $\phi=T \chi_{(0,1)}$ and $\psi=T^{\prime} \chi_{(0,1)}$. It follows that

(i) If $\sigma_{1}$ is a rearrangement invariant norm on $M(0, \infty)$ for which another such norm $\sigma_{2}$ exists with $T \in\left[L^{\sigma_{1}}, L^{\sigma_{2}}\right]$, then $L^{\sigma_{1}} \subset \Lambda(\psi)$.

(ii) If $\sigma_{2}$ is a rearrangement invariant norm on $M(0, \infty)$ for which another such norm $\sigma_{1}$ exists with $T \in\left[L^{\sigma_{1}}, L^{\sigma_{2}}\right]$, then $L^{\sigma_{2}} \supset M(\phi)$. 
Proof. Suppose $f \in L^{\sigma_{1}}$. Then $T f^{*} \in L^{\sigma_{2}}$. As a result, since $\chi_{(0,1)}$ belongs to every rearrangement invariant space,

$$
\int_{0}^{\infty}\left(T f^{*}\right)(t) \chi_{(0,1)}(t) d t<\infty
$$

or, equivalently,

$$
\int_{0}^{\infty} f^{*}(t) \psi(t) d t<\infty .
$$

$T \in\left[L^{\sigma_{1}}, L^{\sigma_{2}}\right]$ implies $T^{\prime} \in\left[L^{\sigma_{2}^{\prime}}, L^{\sigma_{1}{ }^{\prime}}\right]$. By (i), $L^{\sigma_{2}^{\prime} \subset \Lambda(\phi), ~ o r ~ e q u i v a l e n t l y, ~}$ $L^{\sigma_{2}} \supset M(\phi)$.

THEOREM 2.2. Let $T$ be a positive operator of convolution-type having kernel $a(t)$ for which

$$
\int_{0}^{\infty} \min [1,1 / u] a(u) d u<\infty .
$$

Then the function $\phi=T_{\chi_{(0,1)}}$ is nonnegative and nonincreasing on $(0, \infty)$ with $\int_{0}^{1} \phi(t) d t<\infty$. Moreover, to each rearrangement invariant norm $\sigma$ on $M(0, \infty)$ with $L^{\sigma} \supset M(\phi)$ there corresponds a rearrangement invariant norm $\sigma$ such that $T \in\left[L^{\sigma}, L^{\sigma}\right]$.

Proof. Observe that (2.5) is simply the condition that $\Phi(1)=\int_{0}^{1} \phi(t) d t$ be finite. This means that $\Phi(t)$ and hence $\left\|\chi_{(0, t)}\right\|_{M(\phi)}=t / \Phi(t)$ will be finite for all $t>0$.

Given nonnegative $f \in M(0, \infty)$, define $\boldsymbol{\sigma}(f)$ by

$$
\boldsymbol{\sigma}(f)=\sigma\left(T f^{*}\right) .
$$

We show $\boldsymbol{\sigma}$ satisfies the definitive properties of a rearrangement invariant norm given in [4]. In what follows, $f, f_{n}$, and $g$ are nonnegative functions in $M(0, \infty)$.

Now, $\sigma\left(T f^{*}\right) \geq 0$ with equality if and only if

$$
\int_{0}^{\infty} a(s) f^{*}(s t) d s=0, \text { a.e. }
$$

or, equivalently,

$$
\int_{0}^{\infty} a(s / t) f^{*}(s) d s=0, \quad \text { a.e. }
$$

The assumption that $f=0$ a.e. is false ensures the existence of $s_{0}>0$ such that $f^{*}(s)>0$ when $0<s<s_{0}$. But, for all sufficiently small $t$, the function $a(s / t)$ is greater than zero on a subset of $\left(0, s_{0}\right)$ of positive Lebesgue measure. Hence $f=0$ a.e. 
The subadditivity of $\boldsymbol{\sigma}$ will follow by duality given

$$
\int_{0}^{\infty} h(t)\left[T(f+g)^{*}\right](t) d t \leq \sigma\left(T f^{*}\right)+\delta\left(T g^{*}\right)
$$

for all nonnegative, nonincreasing $h \in L^{\sigma^{\prime}}$ with $\sigma^{\prime}(h) \leq 1$. But, the first term in (2.9) is equal to

$$
\int_{0}^{\infty} a(u) d u \int_{0}^{\infty} h(t)(f+g)^{*}(u t) d t
$$

Further,

$$
\int_{0}^{t}(f+g)^{*}(u s) d s \leq \int_{0}^{t} f^{*}(u s) d s+\int_{0}^{t} g^{*}(u s) d s
$$

together with a well-known result of Hardy and Littlewood, ensures (2.10) is dominated by

$$
\int_{0}^{\infty} a(u) d u \int_{0}^{\infty} h(t) f^{*}(u t) d t+\int_{0}^{\infty} a(u) d u \int_{0}^{\infty} h(t) g^{*}(u t) d t .
$$

After inverting the order of the integrals in (2.12), an appeal to the generalized Hölder inequality will yield (2.9).

To verify $\boldsymbol{\sigma}$ satisfies the Fatou property, observe that $0 \leq f_{n} \uparrow f$ implies $T f_{n}^{*} \uparrow T f^{*}$ and hence, by the corresponding property of $\sigma, \boldsymbol{\sigma}\left(f_{n}\right) \uparrow \boldsymbol{\sigma}(f)$.

At this point we obtain from $[9$, p. 42] that $\sigma$ gives rise to a Banach space in the usual way.

Suppose now $E \in \mathfrak{M}$, the class of Lebesgue-measurable subsets of $(0, \infty)$, and that $m(E)<\infty$. Then $T \chi_{E}^{*}$ will belong to $L^{\sigma}$ if there exists $c>0$ so that

$$
\int_{0}^{s}\left[T \chi_{(0, m(E))}\right](u) d u \leq c \int_{0}^{s}\left[T \chi_{(0,1)}\right](u) d u
$$

for all $s>0$. But, (2.13) just asks that

$$
m(E) \Phi(s / m(E)) \leq c \Phi(s) \quad s>0,
$$

which is true with $c=\max (1, m(E))$, since $\Phi$ increases concavely from $\Phi(0)=0$.

Finally, we show that to each $E \in \mathfrak{M}, m(E)<\infty$, there is associated a constant $k_{E}>0$ so that

$$
\int_{E} f(t) d t \leq k_{E} \boldsymbol{\sigma}(f)
$$

for all nonnegative $f \in M(0, \infty)$. It will be enough to show that for such $f$ there is a $k>0$ for which $\sigma(f) \leq k \boldsymbol{\sigma}(f)$, because $\sigma$ satisfies (2.15). To this end, fix $f, g$ and suppose $\sigma^{\prime}(g) \leq 1$. Also, let $u>0$ be such that $A(u) \equiv \int_{0}^{u} a(s) d s>0$. We 
have

$$
\int_{0}^{\infty} 2 f^{*}(t) g^{*}(t) d t \leq \max (1, u) \int_{0}^{\infty} f^{*}(u t) g^{*}(t) d t
$$

since $f^{*}(t) \leq f^{*}(u t)$ for $0<u \leq 1$, while $g^{*}(t) \leq g^{*}(t / u)$ for $u>1$. Now, from Lemma 3.3 of [3],

$$
A(u) \int_{0}^{\infty} f^{*}(u t) g^{*}(t) d t \leq \int_{0}^{u} a(s) d s \int_{0}^{\infty} f^{*}(s t) g^{*}(t) d t
$$

the latter being no bigger than

$$
\begin{aligned}
\int_{0}^{\infty} a(s) d s \int_{0}^{\infty} f^{*}(s t) g^{*}(t) d t & =\int_{0}^{\infty}\left(T f^{*}\right)(t) g^{*}(t) d t \\
& \leq \sigma\left(T f^{*}\right) \sigma^{\prime}\left(g^{*}\right)=\boldsymbol{\sigma}(f)
\end{aligned}
$$

Thus,

$$
\int_{0}^{\infty} f^{*}(t) g^{*}(t) d t \leq k \boldsymbol{\sigma}(f)
$$

where $k=\max (1, u)[A(u)]^{-1}$. The argument is completed on taking the supremum over $g$.

Clearly, $T \in\left[L^{\boldsymbol{\sigma}}, L^{\boldsymbol{\sigma}}\right]$ by the very definition of $\boldsymbol{\sigma}$.

The result dual to Theorem 2.2 is

THEOREM 2.2'. Let $T$ be a positive operator of convolution-type having kernel $a(t)$ and associate $T^{\prime}$. Suppose

$$
\int_{0}^{\infty} \min (1,1 / u) a(1 / u) \frac{d u}{u}<\infty .
$$

Then the function $\psi=T^{\prime} \chi_{(0,1)}$ is nonnegative and nonincreasing on $(0, \infty)$ with $\int_{0}^{1} \psi(t) d t<\infty$. Moreover, to each rearrangement invariant norm $\sigma$ on $M(0, \infty)$ with $L^{\sigma} \subset \Lambda(\psi)$ there corresponds a rearrangement invariant norm $\tilde{\sigma}$ such that $T \in\left[L^{\sigma}, L^{\tilde{\sigma}}\right]$.

Proof. Condition (2.20) is just condition (2.5) of Theorem 2.2 for $T^{\prime}$ and its kernel $(1 / t) a(1 / t)$. Further, $L^{\sigma} \subset \Lambda(\psi)$ implies $L^{\sigma^{\prime}} \supset M(\psi)$. Let $\sigma^{\prime}$ be the norm guaranteed by Theorem 2.2 for $T^{\prime}$ and $\sigma^{\prime}$. Take $\tilde{\sigma}=\left(\boldsymbol{\sigma}^{\prime}\right)^{\prime}$.

Remarks. 1. One may give $\tilde{\sigma}$ a somewhat more explicit form in Theorem $2.2^{\prime}$ using a construction analogue to that in Bennett [2]. Thus, firstly, $\tilde{\sigma}^{0}$ is defined at nonnegative $g \in M(0, \infty)$ by

$$
\tilde{\sigma}^{0}(g)=\inf \left\{\sigma(|f|): g^{* *} \leq\left(T f^{*}\right)^{* *}, f \in L^{\sigma}\right\},
$$

with the convention that $\tilde{\sigma}^{0}(g)=\infty$ if no such $f$ exists. Then, $\tilde{\sigma}$ is given at 
nonnegative $g \in M(0, \infty)$ by

$$
\tilde{\sigma}(g)=\sup \tilde{\sigma}^{0}\left(g \chi_{E}\right),
$$

the supremum being taken over all Lebesgue-measurable subsets $E$ of $(0, \infty)$ with $m(E)<\infty$.

2. It is clear from the constructions of $\boldsymbol{\sigma}$ and $\tilde{\sigma}$ that, with respect to $T, L^{\boldsymbol{\sigma}}$ is the largest domain space having $L^{\sigma}$ as range, while $L^{\tilde{\sigma}}$ is the smallest range space having $L^{\sigma}$ as domain. Further, $L^{\sigma} \subset L^{\sigma}$ and hence $L^{\sigma} \subset L^{\tilde{\sigma}}$. In particular, if $T \in\left[L^{\sigma}\right]$, then $L^{\sigma}=L^{\tilde{\sigma}}=L^{\sigma}$, the norms being equivalent.

DEFINITION 2.1. Let $\sigma_{1}$ and $\sigma_{2}$ be rearrangement invariant norms. The functional $\sigma_{1} \wedge \sigma_{2}$ is given at nonnegative $f \in M(0, \infty)$ by

$$
\left(\sigma_{1} \wedge \sigma_{2}\right)(f)=\max \left[\sigma_{1}(f), \sigma_{2}(f)\right]
$$

REMARK. One readily verifies that $\sigma_{1} \wedge \sigma_{2}$ is a rearrangement invariant norm and that, as sets, $L^{\sigma_{1} \wedge \sigma_{2}}=L^{\sigma_{1}} \cap L^{\sigma_{2}}$. In view of this we will use the intersection notation for $L^{\sigma_{1} \wedge \sigma_{2}}$.

In what follows, $\sigma_{\alpha}$ and $\sigma_{\alpha}^{\prime}(0<\alpha \leq 1)$ will denote the usual Lorentz norms for which $\phi(t)=t^{\alpha-1} ; \Lambda(\alpha), M(\alpha)$ the corresponding Lorentz spaces. To keep notation uniform we will write $\Lambda(0)$ for $L^{\infty}$ and $M(0)$ for $L^{1}$.

THEOREM 2.3. Suppose $T$ is a positive operator of convolution-type with kernel (1.2). Let $\sigma$ denote the usual norm on the Lorentz space $M(\phi), \phi=T \chi_{(0,1)}$. Then, as a set, $L^{\mathbf{\sigma}}$ is equal to $\Lambda\left(p^{-1}\right) \cap \Lambda\left(q^{-1}\right)$. In particular, if $q<\infty$, this is $\Lambda\left(\max \left[t^{\epsilon}, t^{\eta}\right]\right), \epsilon=1 / p-1, \eta=1 / q-1$.

Proof. The boundedness of $T$ follows once it is shown that for $u>0$ a constant multiple of the norm of $f\left(\right.$ in $\Lambda\left(p^{-1}\right) \cap \Lambda\left(q^{-1}\right)$ ) dominates

$$
\int_{0}^{\infty} f^{*}(t) g_{u}(t) d t
$$

where

$$
g_{u}(t)=\left[T^{\prime} \chi_{(0, u)}\right](t) / \Phi(u)
$$

Now, for the kernel $a(r)=\min \left(r^{\epsilon}, r^{\eta}\right)$ one easily sees that

$$
a(r t) \leq \max \left(t^{\epsilon}, t^{\eta}\right) a(r)
$$

and so

$$
g_{u}(t) \leq \int_{t / u}^{\infty} a(r) \frac{d r}{r} / \int_{1 / u}^{\infty} a(r) \frac{d r}{r}
$$


since

$$
\Phi(u)=\int_{0}^{\infty} \min (u, 1 / r) a(r) d r \geq \int_{1 / u}^{\infty} a(r) \frac{d r}{r} .
$$

But,

$$
\int_{1 / u}^{\infty} a(r t) \frac{d r}{r} / \int_{1 / u}^{\infty} a(r) \frac{d r}{r} \leq \max \left(t^{\epsilon}, t^{\mathfrak{S}}\right),
$$

by (2.25). This completes the proof of the boundedness in case $q<\infty$ and, indeed, gives

$$
\int_{1}^{\infty} f^{*}(t) g_{u}(t) d t \leq\|f\|_{\epsilon}
$$

for all $q$. It is enough to show now that

$$
\int_{0}^{1} f^{*}(t) g_{u}(t) d t \leq\|f\|_{\infty} \int_{0}^{1} g_{u}(t) d t=\|f\|_{\infty} .
$$

However,

$$
\int_{0}^{1}\left[T^{\prime} \chi_{(0, u)}\right](t) d t=\int_{0}^{u}\left[T \chi_{(0,1)}\right](t) d t=\Phi(u) .
$$

The methods of [7, Theorem 4.7] readily show $\Lambda\left(p^{-1}\right) \cap \Lambda\left(q^{-1}\right)$ is the largest space having range $M(\phi)$ under $T$. Indeed, suppose, if possible, that $f \in$ $M(0, \infty), T f^{*} \in M(\phi)$, but $f \notin \Lambda\left(p^{-1}\right)$. From

$$
\lim _{u \rightarrow \infty} g_{u}(t)=-\left(1+\epsilon^{-1}\right) t^{\epsilon}
$$

we conclude, using Fatou's lemma on (2.23), that

$$
\|T f\|_{M(\phi)} \geq-\left(1+\epsilon^{-1}\right)\|f\|_{\epsilon}=\infty,
$$

a contradiction. Similar considerations show that when $q<\infty$, one must have $f \in \Lambda\left(q^{-1}\right)$ whenever $T f^{*} \in M(\phi)$. Assume, then, if possible, that $f \in M(0, \infty)$, $\left(P_{\mathrm{p}}+Q_{\infty}\right) f^{*} \in M(\phi)$, but $f \notin L^{\infty}$. Given $B>0$ there must exist $b>0$ such that $f^{*}(t) \geq B$ when $0<t \leq b$. For $u \leq t \leq b$, the expression (2.23) is no smaller than

$$
B(u \ln (b / u))
$$

which approaches $B$ as $u \rightarrow 0+$. Since $B$ was arbitrary, a contradiction has been reached.

In view of the second remark following Theorem $2.2^{\prime}$, the proof is complete.

THEOREM 2.3'. Supose $T$ is a positive operator of convolution-type with kernel (1.2). Let $\sigma$ denote the usual norm on the Lorentz space $\Lambda(\psi), \psi=T^{\prime} \chi_{(0,1)}$. Then, as a set, $L^{\tilde{\sigma}}$ is equal to $M(1-1 / p)+M(1-1 / q)$. In particular, if $p>1$, it is $M\left(\max \left[t^{-\mathbf{p}^{-1}}, t^{-\mathbf{q}^{-1}}\right]\right)$. 
REMARKS. 1. The mappings of Theorems 2.2 and $2.2^{\prime}$ need not invert one another. Thus, if $-1<\eta<\epsilon<0$, Theorem 2.2 shows that both $L^{1} \cap L^{\infty}$ and $\Lambda\left(p^{-1}\right) \cap \Lambda\left(q^{-1}\right)\left(L^{1} \cap L^{\infty} \mp \Lambda\left(p^{-1}\right) \cap \Lambda\left(q^{-1}\right)\right)$ must have $M(\phi)$ as their minimal range space under $T$. The other assertion follows by duality.

The above example leads to the conjecture that the mappings of Theorems 2.2 and $2.2^{\prime}$ applied succesively to $L^{\sigma} \cap \Lambda(\psi)$ yield the space $\left(L^{\sigma} \cap \Lambda(\psi)\right)+L^{\rho}$, $\rho$ being the usual norm of $M(\phi)$. This would just be $L^{\sigma}$ when that space is intermediate between $\Lambda\left(p^{-1}\right)$ and $\Lambda\left(q^{-1}\right)$; that is,

$$
\Lambda\left(p^{-1}\right) \cap \Lambda\left(q^{-1}\right) \subset L^{\sigma} \subset \Lambda\left(p^{-1}\right)+\Lambda\left(q^{-1}\right) .
$$

In view of [4, Lemma 2] and [1, Theorem 13.VII], then the conjecture would not hold if $L^{\sigma}$ were intermediate but not such that all operators in $\left[\Lambda\left(p^{-1}\right) \cap\right.$ $\left[\Lambda\left(q^{-1}\right)\right]$ were in $\left[L^{\sigma}\right]$. But, for $p=2, q=4, L^{\sigma} \equiv L^{2} \cap \Lambda\left(\frac{1}{3}\right)$ satisfies (2.35), while the mapping that sends $f$ to

$$
\left(\int_{0}^{\infty} \min \left(t^{-1 / 2}, t^{-3 / 4}\right) f(t) d t\right) \chi_{(0,1)}
$$

is in $[\Lambda(1 / 2)] \cap[\Lambda(1 / 4)]$, though not in $\left[L^{\sigma}\right]$.

2. It is easily seen that the mappings of Theorems 2.2 and $2.2^{\prime}$ do invert each other when restricted in the domain spaces to the $L^{\sigma}$ or in the range spaces to the $L^{\tilde{\sigma}}$.

3. If $T$ has kernel

$$
\max \left[t^{1 / p-1}, t^{1 / q-1}\right], \quad 1<p \leq q<\infty,
$$

then it will be bounded between every reasonable pair of rearrangement invariant spaces; more precisely,

$$
T \in[\Lambda(\psi), M(\phi)] .
$$

Indeed, (2.38) will be true for a general $T$ of form (1.1) if and only if

$$
\Phi(r s) \leq c \Phi(r) \Phi(s)
$$

$c>0$ being independent of $r, s>0$.

\section{REFERENCES}

1. N. Aronszajn and E. Gagliardo, Interpolation spaces and interpolation methods, Ann. Mat. Pura. Appl., 68 (1965), 51-118.

2. C. Bennett, A Hausdorf-Young theorem for rearrangement-invariant spaces, Pac. J. Math., 47 (1973), 311-328.

3. D. W. Boyd, The Hilbert transform on rearrangement-invariant spaces, Canad. J. Math., 19 (1967), 599-616.

4. _ Indices of function spaces and their relationship to interpolation, Canad. J. Math., 21 (1969), 1245-1254.

5. _ Spectra of convolution operators, Acta. Sci. Math. (Szeged), 35 (1973), 31-37. 
6. A. P. Calderón, Spaces between $L^{1}$ and $L^{\infty}$ and the theorem of Marcinkiewicz, Studia Math. 26 (1966), 273-299.

7. R. A. Kerman, Generalized Hankel conjugate transformations on rearrangement invariant spaces, Trans. Amer. Math Soc., 232 (1977), 111-130.

8. - Optimal pairs of function spaces associated by operators of weak-type, Manuscript.

9. W. A. J. Luxemburg and A. C. Zaanen, Riesz Spaces I, North-Holland Pub. Co., 1971.

DEPARTMENT OF MATHEMATICS

BROCK UNIVERSTTY

St. Catharines, Ontario, L2S 3A1 